\title{
Parameter Derivatives of the Jacobi Polynomials with Three Variables on the Simplex
}

\author{
Rabia Aktaş \\ Ankara University, Faculty of Science, Department of Mathematics, 06100, Ankara, Turkey
}

\begin{abstract}
In this paper, an attempt has been made to derive parameter derivatives of Jacobi polynomials with three variables on the simplex. They are obtained via parameter derivatives of the classical Jacobi polynomials $P_{n}^{(\alpha, \beta)}(x)$ with respect to their parameters. The study is motivated by the expansions of parameter derivatives which are obtained for the classical Jacobi polynomials over the interval $(-1,1)$, Jacobi polynomials with two variables on the triangle and some other families of orthogonal polynomials in two variables.
\end{abstract}

\section{Introduction}

In literature, there are many studies on the parameter derivatives of various special functions which find applications in applied mathematics, several branches of mathematics, mathematical and theoretical physics. In $[13,14]$, author has studied the derivative of the Legendre function of the first kind, with respect to its degree $v,\left[\partial P_{v}(z) / \partial v\right]_{v=n}(n \in \mathbb{N})$, and has obtained its some representations, which are encountered in some problems such as in the general theory of relativity and in solving some boundary value problems of potential theory, of electromagnetism and of heat conduction in solids. The derivatives of the associated Legendre function of the first kind with respect to its order and its degree (with applications to the construction of the associated Legendre function of the second kind of integer degree and order) have been obtained in $[15,16]$. These derivatives of the associated Legendre function arise in solutions of some problems of heat conduction, theoretical acoustics and other branches of theoretical physics.

To obtain parameter derivative representations for orthogonal polynomials with one variable, a general method has been investigated in the following form

$$
\frac{\partial P_{n}(\lambda ; x)}{\partial \lambda}=\sum_{k=0}^{n} c_{n, k}(\lambda) P_{k}(\lambda ; x)
$$

for orthogonal polynomials with variable $x$, with $\lambda$ being a parameter, and such derivative representations have been obtained by many authors for orthogonal polynomials in one variable $[6,7,8,9,11,12,17]$. In particular, the explicit expansions of the form (1) have been investigated for dicrete Laguerre polynomials in [17], for Jacobi polynomials in [6], for Gegenbauer polynomials and the generalized Laguerre polynomials in [7]. The following representation given in [7] for the generalized Laguerre polynomials

$$
\frac{\partial L_{n}^{(\alpha)}(x)}{\partial \alpha}=\sum_{k=0}^{n-1} \frac{1}{n-k} L_{k}^{(\alpha)}(x)
$$

has applications in relativistic quantum mechanics. Also, the parameter derivative representations in the form of (1) for all the continuous and discrete orthogonal polynomials have been studied in [8]. Moreover, in [11], the following expansion form which is general than (1)

$$
\frac{\partial^{m} P_{n}(\lambda ; x)}{\partial \lambda^{m}}=\sum_{k=0}^{n} a_{n, k}(m, \lambda) P_{k}(\lambda ; x) \quad(m \in \mathbb{N})
$$

has been investigated and the recurrence relations for coefficients in the expansion has been obtained. In [9], a way to obtain parameter derivative representations of order $m \quad(m \in \mathbb{N})$ for the families of almost all the classical orthogonal polynomials has been studied.

The representations of parameter derivatives for Jacobi polynomials $P_{n}^{(\alpha, \beta)}(x)$ obtained by Froehlich are as follows

$$
\begin{aligned}
\frac{\partial P_{n}^{(\alpha, \beta)}(x)}{\partial \alpha} & =[\psi(2 n+\alpha+\beta+1)-\psi(n+\alpha+\beta+1)] P_{n}^{(\alpha, \beta)}(x) \\
& +\sum_{k=0}^{n-1} \frac{(2 k+\alpha+\beta+1) \Gamma(k+\alpha+\beta+1)}{(n-k)(n+k+\alpha+\beta+1) \Gamma(k+\beta+1)} \\
& \times \frac{\Gamma(n+\beta+1)}{\Gamma(n+\alpha+\beta+1)} P_{k}^{(\alpha, \beta)}(x)
\end{aligned}
$$

and 


$$
\begin{aligned}
& \frac{\partial P_{n}^{(\alpha, \beta)}(x)}{\partial \beta} \\
& =[\psi(2 n+\alpha+\beta+1)-\psi(n+\alpha+\beta+1)] P_{n}^{(\alpha, \beta)}(x) \\
& +\sum_{k=0}^{n-1} \frac{(-1)^{n+k}(2 k+\alpha+\beta+1) \Gamma(k+\alpha+\beta+1)}{(n-k)(n+k+\alpha+\beta+1) \Gamma(k+\alpha+1)} \\
& \times \frac{\Gamma(n+\alpha+1)}{\Gamma(n+\alpha+\beta+1)} P_{k}^{(\alpha, \beta)}(x)
\end{aligned}
$$

for $\alpha, \beta>-1$ where $\psi(\xi)=\frac{1}{\Gamma(\xi)} \frac{d \Gamma(\xi)}{d \xi}$ is the digamma function, which satisfies

$[\psi(\alpha+n)-\psi(\alpha)]=\sum_{k=0}^{n-1} \frac{1}{\alpha+k}$

(see ([1], ? 6.3 ), [7]).

We remind that the classical Jacobi polynomials $P_{n}^{(\alpha, \beta)}(x)$ which are orthogonal polynomials with respect to the inner product

$$
\langle f, g\rangle_{\alpha, \beta}:=\int_{-1}^{1} f(x) g(x)(1-x)^{\alpha}(1+x)^{\beta} d x
$$

are defined through

$$
\begin{aligned}
P_{n}^{(\alpha, \beta)}(x)= & { }_{2} F_{1}\left[-n, \alpha+\beta+n+1 ; \alpha+1 ; \frac{1-x}{2}\right] \\
& \times \frac{(\alpha+1)_{n}}{n !}
\end{aligned}
$$

where ${ }_{2} F_{1}$ denotes Gauss hypergeometric function and the Pochammer symbol is defined by

$$
(\alpha)_{0}=1, \quad(\alpha)_{k}=\frac{\Gamma(\alpha+k)}{\Gamma(\alpha)}, k=1,2, \ldots .
$$

In the recent papers [2, 3, 4], author has studied similar expansion in the form of

$$
\begin{aligned}
\frac{\partial P_{n, k}(\lambda ; x, y)}{\partial \lambda}= & \sum_{m=0}^{n-1} \sum_{j=0}^{m} d_{n, j, m} P_{m, j}(\lambda ; x, y) \\
& +\sum_{j=0}^{k} e_{n, j, k} P_{n, j}(\lambda ; x, y)
\end{aligned}
$$

for orthogonal polynomials with two variables, with $\lambda$ being a parameter and $0 \leq k \leq n ; n \in \mathbb{N}_{0}$ and has given parameter derivative representations for Jacobi polynomials with two variables on the triangle and some families of orthogonal polynomials in two variables by using the expansions (2) and (3) given for Jacobi polynomials with one variable. Motivated and inspired by the works $[2,3,4,6,7,8,9,11,12,17]$, we derive representations of parameter derivatives for Jacobi polynomials with three variables on the simplex similar to the expansions in the forms of (1) and (4) by using the parametric derivatives of the classical Jacobi polynomials $P_{n}^{(\alpha, \beta)}(x)$.

\section{The parameter derivatives of Jacobi polynomials with three variables on the simplex}

The Jacobi polynomials with three variables on the simplex

$$
T^{3}:=\left\{(x, y, z) \in \mathbb{R}^{3}: x, y, z \geq 0,1-x-y-z \geq 0\right\}
$$

are orthogonal with respect to the weight function $W_{\alpha, \beta, \gamma, \delta}(x, y, z)=x^{\alpha} y^{\beta} z^{\gamma}(1-x-y-z)^{\delta} \quad$ for $\alpha, \beta, \gamma, \delta>-1$ with respect to the inner product

$\langle f, g\rangle_{\alpha, \beta, \gamma, \delta}:=\int_{T^{3}} f(x, y, z) g(x, y, z) W_{\alpha, \beta, \gamma, \delta}(x, y, z) d x d y d z$

Let $V_{n}^{3}\left(W_{\alpha, \beta, \gamma, \delta}\right)$ be the space of polynomials of degree $n$ which are orthogonal with respect to this inner product. One of the bases for $V_{n}^{3}\left(W_{\alpha, \beta, \gamma, \delta}\right)$ is as follows

$$
\begin{aligned}
& P_{m, k}^{n}(x, y, z):=P_{n, m, k}^{(\alpha, \beta, \gamma, \delta)}(x, y, z) \\
& =P_{n-m}^{(2 m+\beta+\gamma+\delta+2, \alpha)}(2 x-1)(1-x)^{m-k} P_{m-k}^{(2 k+\gamma+\delta+1, \beta)}\left(\frac{2 y}{1-x}-1\right) \\
& \times(1-x-y)^{k} P_{k}^{(\delta, \gamma)}\left(\frac{2 z}{1-x-y}-1\right)
\end{aligned}
$$

for $0 \leq k \leq m \leq n ; n \in \mathbb{N}_{0}$ where $P_{n}^{(\alpha, \beta)}(x)$ denotes the classical Jacobi polynomial of degree $n$ ( see [5]).

Since the simplex $T^{3}$ is symmetric under the permutation of $(x, y, z, 1-x-y-z) \quad$, by parametrizing the simplex differently, we can give the following orthogonal bases of $V_{n}^{3}\left(W_{\alpha, \beta, \gamma, \delta}\right)$

$$
\begin{aligned}
& T_{m, k}^{n}(x, y, z):=P_{n, m, k}^{(\beta, \gamma, \alpha, \delta)}(y, z, x), \\
& S_{m, k}^{n}(x, y, z):=P_{n, m, k}^{(\gamma, \alpha, \beta, \delta)}(z, x, y)
\end{aligned}
$$

and

$$
Q_{m, k}^{n}(x, y, z):=P_{n, m, k}^{(\delta, \beta, \gamma, \alpha)}(1-x-y-z, y, z)
$$

for $0 \leq k \leq m \leq n ; n \in \mathbb{N}_{0}$. These bases of $V_{n}^{3}\left(W_{\alpha, \beta, \gamma, \delta}\right)$ are given explicitly by

$$
\begin{aligned}
T_{m, k}^{n}(x, y, z) & =P_{n-m}^{(2 m+\alpha+\gamma+\delta+2, \beta)}(2 y-1)(1-y)^{m-k} \\
& \times P_{m-k}^{(2 k+\alpha+\delta+1, \gamma)}\left(\frac{2 z}{1-y}-1\right)(1-y-z)^{k} \\
& \times P_{k}^{(\delta, \alpha)}\left(\frac{2 x}{1-y-z}-1\right)
\end{aligned}
$$




$$
\begin{aligned}
S_{m, k}^{n}(x, y, z) & =P_{n-m}^{(2 m+\alpha+\beta+\delta+2, \gamma)}(2 z-1)(1-z)^{m-k} \\
& \times P_{m-k}^{(2 k+\beta+\delta+1, \alpha)}\left(\frac{2 x}{1-z}-1\right)(1-x-z)^{k} \\
& \times P_{k}^{(\delta, \beta)}\left(\frac{2 y}{1-x-z}-1\right)
\end{aligned}
$$

and

$$
\begin{aligned}
Q_{m, k}^{n}(x, y, z) & =P_{n-m}^{(2 m+\alpha+\beta+\gamma+2, \delta)}(1-2 x-2 y-2 z) \\
& \times(x+y+z)^{m-k} P_{m-k}^{(2 k+\alpha+\gamma+1, \beta)}\left(\frac{2 y}{x+y+z}-1\right) \\
& \times(x+z)^{k} P_{k}^{(\alpha, \gamma)}\left(\frac{2 z}{x+z}-1\right),
\end{aligned}
$$

for $0 \leq k \leq m \leq n ; n \in \mathbb{N}_{0}$ (see [5]).

Now, by using the parameter derivative representation given by (3), let's obtain similar representations for Jacobi polynomials with three variables on the simplex.

Theorem 1. For $\quad \alpha, \beta, \gamma, \delta>-1 \quad$, $0 \leq k \leq m \leq n ; n \in \mathbb{N}_{0} \quad$, Jacobi polynomials $P_{m, k}^{n}(x, y, z)$ satisfy the following representation for $n \geq m+1, \quad m \geq k \geq 0$

$$
\begin{aligned}
& \frac{\left.\partial P_{m, k}^{n} x, y, z\right)}{\partial \alpha} \\
& =[\psi(2 n+\alpha+\beta+\gamma+\delta+3)-\psi(n+m+\alpha+\beta+\gamma+\delta+3)] P_{m, k}^{n}(x, y, z) \\
& +\frac{\Gamma(n+m+\beta+\gamma+\delta+3)}{\Gamma(n+m+\alpha+\beta+\gamma+\delta+3)} \\
& \times \sum_{s=0}^{n-m-1} \frac{(-1)^{s+1} \Gamma(n+m-s+\alpha+\beta+\gamma+\delta+2)}{\Gamma(n+m-s+\beta+\gamma+\delta+2)} \\
& \times \frac{(2 n-2 s+\alpha+\beta+\gamma+\delta+1)}{(s+1)(2 n-s+\alpha+\beta+\gamma+\delta+2)} P_{m, k}^{n-s-1}(x, y, z) \\
& \text { and for } n=m \geq k \geq 0 \\
& \frac{\partial P_{n, k}^{n}(x, y, z)}{\partial \alpha}=0 .
\end{aligned}
$$

Proof. If we take derivative of the Jacobi polynomials with three variables $P_{m, k}^{n}(x, y, z)$ with respect to its parameter $\alpha$ and then take into consideration the relation (3), we find the desired result.

Similarly, one can easily obtain the following representations for the other bases $T_{m, k}^{n}(x, y, z), S_{m, k}^{n}(x, y, z)$ and $Q_{m, k}^{n}(x, y, z)$.

Theorem 2. For $\alpha, \beta, \gamma, \delta>-1$, we get the next results for $n \geq m+1, \quad m \geq k \geq 0$

$$
\begin{aligned}
& \frac{\partial T_{m, k}^{n}(x, y, z)}{\partial \beta} \\
& =[\psi(2 n+\alpha+\beta+\gamma+\delta+3)-\psi(n+m+\alpha+\beta+\gamma+\delta+3)] T_{m, k}^{n}(x, y, z) \\
& +\frac{\Gamma(n+m+\alpha+\gamma+\delta+3)}{\Gamma(n+m+\alpha+\beta+\gamma+\delta+3)} \\
& \times \sum_{s=0}^{n-m-1} \frac{(-1)^{s+1} \Gamma(n+m-s+\alpha+\beta+\gamma+\delta+2)}{\Gamma(n+m-s+\alpha+\gamma+\delta+2)} \\
& \times \frac{(2 n-2 s+\alpha+\beta+\gamma+\delta+1)}{(s+1)(2 n-s+\alpha+\beta+\gamma+\delta+2)} T_{m, k}^{n-s-1}(x, y, z), \\
& \frac{\partial S_{m, k}^{n}(x, y, z)}{\partial \gamma} \\
& =[\psi(2 n+\alpha+\beta+\gamma+\delta+3)-\psi(n+m+\alpha+\beta+\gamma+\delta+3)] S_{m, k}^{n}(x, y, z) \\
& +\frac{\Gamma(n+m+\alpha+\beta+\delta+3)}{\Gamma(n+m+\alpha+\beta+\gamma+\delta+3)} \\
& \times \sum_{s=0}^{n-m-1} \frac{(-1)^{s+1} \Gamma(n+m-s+\alpha+\beta+\gamma+\delta+2)}{\Gamma(n+m-s+\alpha+\beta+\delta+2)} \\
& \times \frac{(2 n-2 s+\alpha+\beta+\gamma+\delta+1)}{(s+1)(2 n-s+\alpha+\beta+\gamma+\delta+2)} S_{m, k}^{n-s-1}(x, y, z),
\end{aligned}
$$

and

$\frac{\partial Q_{m, k}^{n}(x, y, z)}{\partial \delta}$

$=[\psi(2 n+\alpha+\beta+\gamma+\delta+3)-\psi(n+m+\alpha+\beta+\gamma+\delta+3)] Q_{m, k}^{n}(x, y, z)$

$+\frac{\Gamma(n+m+\alpha+\beta+\gamma+3)}{\Gamma(n+m+\alpha+\beta+\gamma+\delta+3)}$

$\times \sum_{s=0}^{n-m-1} \frac{(-1)^{s+1} \Gamma(n+m-s+\alpha+\beta+\gamma+\delta+2)}{\Gamma(n+m-s+\alpha+\beta+\gamma+2)}$

$\times \frac{(2 n-2 s+\alpha+\beta+\gamma+\delta+1)}{(s+1)(2 n-s+\alpha+\beta+\gamma+\delta+2)} Q_{m, k}^{n-s-1}(x, y, z)$.

For $n=m \geq k \geq 0$,

$$
\frac{\partial T_{n, k}^{n}(x, y, z)}{\partial \beta}=\frac{\partial S_{n, k}^{n}(x, y, z)}{\partial \gamma}=0
$$

and

$$
\frac{\partial Q_{n, k}^{n}(x, y, z)}{\partial \delta}=0 .
$$

\section{Conclusion}

This paper is motivated by the works on the representations of parameter derivatives for orthogonal polynomials with one variable and two variables. In this study, we give parameter expansions for Jacobi polynomials with three variables on simplex, which such parameter derivatives are derived via the expansion (3) obtained by means of the parameter derivatives of Gauss Hypergeometric function for the classical Jacobi 
polynomials $P_{n}^{(\alpha, \beta)}(x)$. Also, we investigate explicit parameter derivative representations for the other orthogonal bases on the simplex by parametrizing the simplex differently. Furthermore, the orthogonality properties of such parametric derivatives can be studied in the further research.

\section{References}

1. M. Abramowitz, I. A. Stegun, Handbook of Mathematical Functions. Dover Publ., New York, (1964)

2. R. Aktaş, A note on parameter derivatives of the Jacobi polynomials on the triangle, Appl. Math. and Comp., 247 (2014), 368-372.

3. R. Aktaş, On parameter derivatives of a family of polynomials in two variables, Appl. Math. and Comp., 256 (2015), 769-777.

4. R. Aktaş, Representations for parameter derivatives of some Koornwinder polynomials in two variables,Journal of the Egyptian Mathematical Society, In Press.

5. C. F. Dunkl, Y. Xu, Orthogonal Polynomials of Several Variables. Encyclopedia of Mathematics and its Applications 81. Cambridge: Cambridge University Press, (2001)

6. J. Froehlich, Parameter derivatives of the Jacoby polynomials and the gaussian hypergeometric function, Integral Transforms Spec. Funct., 2(4) (1994), 253-266.

7. W. Koepf, Identities for families of orthogonal polynomials and special functions, Integral Transforms Spec. Funct., 5(1-2) (1997), 69-102.

8. W. Koepf, D. Schmersau, Representations of orthogonal polynomials, J. Comput. Appl. Math., 90 (1998), 57-94.
9. S. Lewanowicz, Representations for the parameter derivatives of the classical orthogonal polynomials , Rend. Circ. Mat. Palermo, Ser. II, Suppl. 68 (2002), 599-613.

10. E.D. Rainville, Special Functions, The Macmillan Co., New York, (1960)

11. A. Ronveaux, A. Zarzo, I. Area, E. Godoy, Classical orthogonal polynomials: dependence on parameters,J.Comput. Appl. Math., 121 (2000), 95112.

12. R. Szmytkowski, A note on parameter derivatives of classical orthogonal polynomials, arXiv: $0901.2639 \mathrm{v} 3$.

13. R. Szmytkowski, On the derivative of the Legendre function of the first kind with respect to its degree, J. Phys. A, 39 (2006), 15147-15172 [corrigendum: J. Phys. A, 40 (2007), 7819-7820].

14. R. Szmytkowski, Addendum to 'On the derivative of the Legendre function of the first kind with respect to its degree', J. Phys. A, 40 (2007), 14887-14891.

15. Szmytkowski, On the derivative of the associated Legendre function of the first kind of integer degree with respect to its order (with applications to the construction of the associated Legendre function of the second kind of integer degree and order), J. Math. Chem., 46 (2009), 231-260.

16. Szmytkowski, On the derivative of the associated Legendre function of the first kind of integer order with respect to its degree (with applications to the construction of the associated Legendre function of the second kind of integer degree and order), J. Math. Chem., 49 (2011), 1436-1477.

17. M. Wulkow, Numerical treatment of countable systems of ordinary differential equations, KonradZuse-Zentrum Berlin, Techn. Rep. TR 90-8, (1990) 